\title{
Is there an inherited anatomical conformation favoring aneurysmal formation of the anterior communicating artery?
}

\author{
Romain Bourcier, MD, ${ }^{1-3}$ Cédric Lenoble, MD, ${ }^{1}$ Béatrice Guyomarch-Delasalle, MSc, ${ }^{2,3}$ \\ Benjamin Daumas-Duport, MD, ${ }^{1}$ Chrysanthi Papagiannaki, MD, ${ }^{4}$ Richard Redon, $\mathrm{PhD},{ }^{2,3}$ and \\ Hubert Desal, MD, PhD ${ }^{1-3}$

\begin{abstract}
'Department of Diagnostic and Interventional Neuroradiology, Hospital Guillaume et René Laennec; ${ }^{2}$ INSERM, UMR1087, I'institut du thorax, CHU de Nantes; ${ }^{3}$ CNRS, UMR 6291, Université de Nantes, Nantes; and ${ }^{4}$ Department of Diagnostic and Interventional Neuroradiology, CHU Rouen, Rouen, France
\end{abstract}

\begin{abstract}
OBJECTIVE The pathophysiological mechanisms responsible for the formation of intracranial aneurysms (IAs) remain only partially elucidated. However, current evidence suggests a genetic component. The purpose of this study was to investigate the specific anatomical variations in the arterial complex that are associated with the presence of anterior communicating artery (ACOA) aneurysms in the familial forms of IAs.

METHODS This multicenter study investigated bifurcation IAs in patients who had a sporadic ACOA IA without a family history of IA (SACAA group), in patients who had an ACoA IA with a family history of IA (FACAA group), and in their healthy first-degree relatives (HFDRs). Through the use of MR angiography (MRA) reconstructions, the symmetry of the $A_{1}$ segments and the angle between the $A_{1}$ and $A_{2}$ segments were analyzed on $3 D$ models for each group. These measurements were then compared among the 3 groups.
\end{abstract}

RESULTS Twenty-four patients with SACAA, 24 patients with FACAA, and 20 HFDRs were included in the study. Asymmetrical configuration of the $A_{1}$ segments was more frequent in the FACAA group than in the HFDR group $(p=0.002)$. The aneurysm-side $A_{1}-A_{2}$ angle was lower in the FACAA group $(p=0.003)$ and SACAA group $(p=0.007)$ than in the HFDR group. On the contralateral side, there was no difference in $A_{1}-A_{2}$ angles between groups.

CONCLUSIONS The anatomical shape of the ACOA complex seems to be similarly associated with the presence of ACOA IAs in both the FACAA and SACAA groups. This highlights the role played by hemodynamic constraints in aneurysm formation and questions the hypothesis of the hereditary character of these anatomical shapes.

https://thejns.org/doi/abs/10.3171/2016.4.JNS153032

KEY WORDS aneurysm; genetics; hemodynamics; anterior communicating artery; vascular disorders

$\mathrm{D}$ ETECTION of incidental intracranial aneurysms (IAs) seems to be rising because of the increased accessibility and evolution of imaging techniques. ${ }^{21,31}$ The prevalence of IAs in a middle-aged population is $3 \%{ }^{40}$ Many hypotheses about the acquired risk factors and the hemodynamic stress responsible for triggering their formation and evolution have been proposed but have not been unanimously accepted. ${ }^{13}$
Recently, other factors, such as congenital and genetic abnormalities, have been recognized to play an important role since a family history of IA is the most significant risk factor for an IA diagnosis in any individual. ${ }^{4,36}$ The presence of at least 2 first-degree relatives with IAs is defined as familial form aneurysms, which represent $7 \%-12.5 \%$ of the IA population.., 34

The exact nature of this possible genetic predisposition

ABBREVIATIONS $\mathrm{ACOA}=$ anterior communicating artery; $\mathrm{CTA}=$ computed tomography angiography; $\mathrm{CW}=$ circle of Willis; $\mathrm{FACAA}=$ familial anterior communicating artery aneurysm; FIA = familial intracranial aneurysm; GAIA = genetics of intracranial aneurysm; HFDR = healthy first-degree relative; $I A=$ intracranial aneurysm; MIP = maximum intensity projection; MRA = magnetic resonance angiography; PACS = picture archiving and communication system; SACAA = sporadic anterior communicating artery aneurysm; VAC = vascular asymmetry coefficient.

SUBMITTED December 28, 2015. ACCEPTED April 11, 2016.

INCLUDE WHEN CITING Published online June 17, 2016; DOI: 10.3171/2016.4.JNS153032. 
leading to aneurysm formation has yet to be discovered. ${ }^{35}$ Familial aneurysms are usually detected at specific locations for each family, which assumes a focal pathological process. ${ }^{26}$

The interactions among organ development, angiogenesis, cell-matrix adhesion, and inflammatory or hemodynamic response in this process are as yet unknown. However, in patients with sporadic aneurysms, the effect of specific anatomical variations within the circle of Willis (CW) on the formation of IA has been well established. ${ }^{2,6-8,16}$

The purpose of this study was to investigate specific anatomical variations within the anterior communicating artery (ACoA) complex that may be significantly associated with the presence of IAs. For this purpose we compared anatomical and structural characteristics of the ACoA complex, such as asymmetry and angle measurement, in 3 groups: patients with a familial ACoA aneurysm (FACAA), patients with a sporadic ACoA aneurysm (SACAA), and healthy first-degree relatives (HFDRs) of FACAA patients.

\section{Methods \\ Study Population}

Between April 2013 and June 2015, we prospectively included in our study 68 patients from multiple centers. These patients were divided into 3 groups: 1) patients with an aneurysm occurring within the ACoA or the $\mathrm{A}_{1^{-}}$ $\mathrm{A}_{2}$ junction and at least 1 first-degree relative harboring an IA, i.e., familial (FACAA); 2) first-degree relatives belonging to the same generation as the FACAA patients and having no aneurysm (HFDR); and 3) patients with an aneurysm within the ACoA or $\mathrm{A}_{1}-\mathrm{A}_{2}$ junction without a first-degree relative harboring an IA, i.e., sporadic (SACAA).

We used the following inclusion criteria: 1) all patients had to be age 20 or older; 2) patients with FACAA and SACAA had to have a saccular bifurcation IA in the ACoA and a measured major axis of at least $2 \mathrm{~mm}$; and 3) FACAA patients and their HFDRs had to have at least 2 first-degree relatives (including the FACAA patient) suffering from an IA.

We excluded patients suffering from syndromic diseases known to have IA occurring therein: Marfan syndrome, Type II or IV Ehlers-Danlos syndrome, autosomal dominant polycystic kidney disease, and moyamoya syndrome. Dissecting aneurysms, mycotic aneurysms, or aneurysms associated with arteriovenous malformation were likewise excluded from the study.

Patients with SACAAs were included in the study in the order in which they were admitted to the Diagnostic and Therapeutic Neuroradiology Department of the Nantes University Hospital Center. The recruitment of patients with FACAAs and their HFDRs was performed by the Genetics of Intracranial Aneurysm (GAIA) clinical network in France (Nantes, Angers, Bordeaux, Rennes, Tours, Poitiers, and Créteil). Syndromic disorders and "atypical" IA phenotypes were ruled out by consultation with both a medical geneticist and an experienced interventional neuroradiologist in each recruiting center.
Patient information was gathered on history of smoking, alcohol consumption, and cardiovascular risk factors such as diabetes, high blood pressure, and high cholesterol. Patients were considered hypertensive if they were being treated with an antihypertensive drug or if they had systolic blood pressure of at least $140 \mathrm{~mm} \mathrm{Hg}$ or diastolic blood pressure of at least $90 \mathrm{~mm} \mathrm{Hg}$. Patients were considered diabetic if they were being treated with oral antidiabetic medication or insulin. Patients were considered to have dyslipidemia if they were being treated with a lipidlowering drug or had a history of dyslipidemia.

This project received a favorable ethical rating from the Nantes Health Field Ethics Group (GNEDS) on July 18, 2013. This nonintervention research was approved by the French Committee for Personal Protection (CPP) on July 3,2013 . The protocol was submitted and accepted by the French Advisory Committee on Information Processing in Material Research in the Field of Health (CCTIRS) on August 1,2013, and then by the French National Committee for Data Protection and Liberties (CNIL).

\section{D Model Reconstruction}

Each patient's imaging was performed at the coordinating center (Nantes University Hospital Center) using a Siemens AERA 1.5-T machine (Sonata, Siemens). The 3D reconstructions were generated from images belonging to a time-of-flight MR angiography (MRA) sequence having the following parameters: TE $7 \mathrm{msec}$, TR $24 \mathrm{msec}$, flip angle $20^{\circ}$, FOV $180 \mathrm{~mm}$, slice thickness $0.7 \mathrm{~mm}$. Patients who were recruited to the study from associated recruiting centers had time-of-flight sequences performed on the University Hospital Center machines, which were deemed to be of sufficient quality to be usable and suitable for required measurements.

All of the images were recorded in DICOM format and transferred to a picture archiving and communication system (PACS) station (Carestream Health). The vascular compartment was isolated by automatic thresholding, and the vascular and aneurysmal contours were reconstructed by means of algorithmic smoothing. The 3D surface model of the aneurysm and the vessels of the anterior complex of the CW could then be manipulated freely within the PACS software. Diameters and angles were measured using the vascular study software from the PACS.

\section{Definition of Morphological Parameters}

We bilaterally studied the mean diameter and angles of the vessels within the anterior complex of the $\mathrm{CW}$, including the $A_{1}$ and $A_{2}$ segments. The $A_{1}$ segment begins at the junction of the carotid artery and ends at the junction of the ACoA. The $\mathrm{A}_{2}$ segment begins at the ACoA junction and ends at the rostrum-genu junction of the corpus callosum.

The $\mathrm{A}_{1}$ and $\mathrm{A}_{2}$ segments were automatically reconstructed on a 3D model, making the barycenter of the vessel visible (Figs. 1 and 2). Diameter measurements for the $A_{1}$ segment were performed on a cross-section of the vessel at a point equidistant from the carotid artery and $\mathrm{A}_{1}-\mathrm{A}_{2}$ junctions (Fig. 3). The PACS software automatically calculated maximum and minimum diameters. We used this information to 


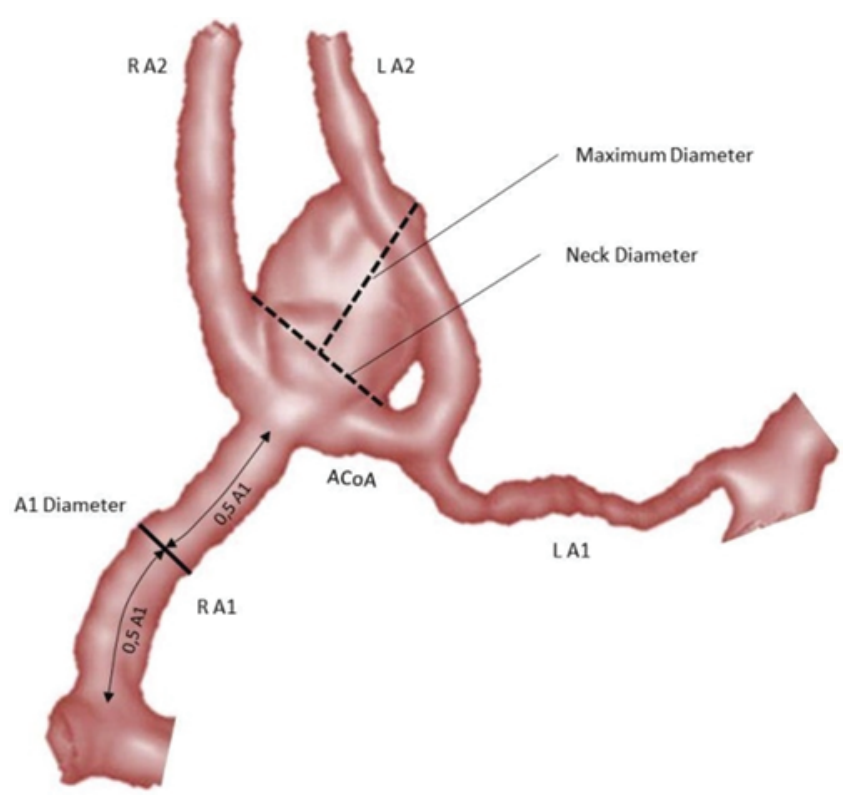

FIG. 1. Three-dimensional model of an $A C o A$ aneurysm, frontal view, showing the diameter measurements. $L=$ left; $R=$ right.

calculate mean diameter, which is necessary for assessing the vascular asymmetry coefficient (VAC). The VAC corresponds to the difference between the mean diameters of the right and left segments and is expressed as the highest mean diameter percentage. When the VAC was less than $10 \%$, the $\mathrm{A}_{1}$ segments were classified as symmetrical. When the VAC was between $10 \%$ and $40 \%$, the $\mathrm{A}_{1}$ segments were classified as asymmetrical. When the VAC was greater than $40 \%$, the $\mathrm{A}_{1}$ segments were classified as hypoplastic. The $\mathrm{A}_{1}$ segment

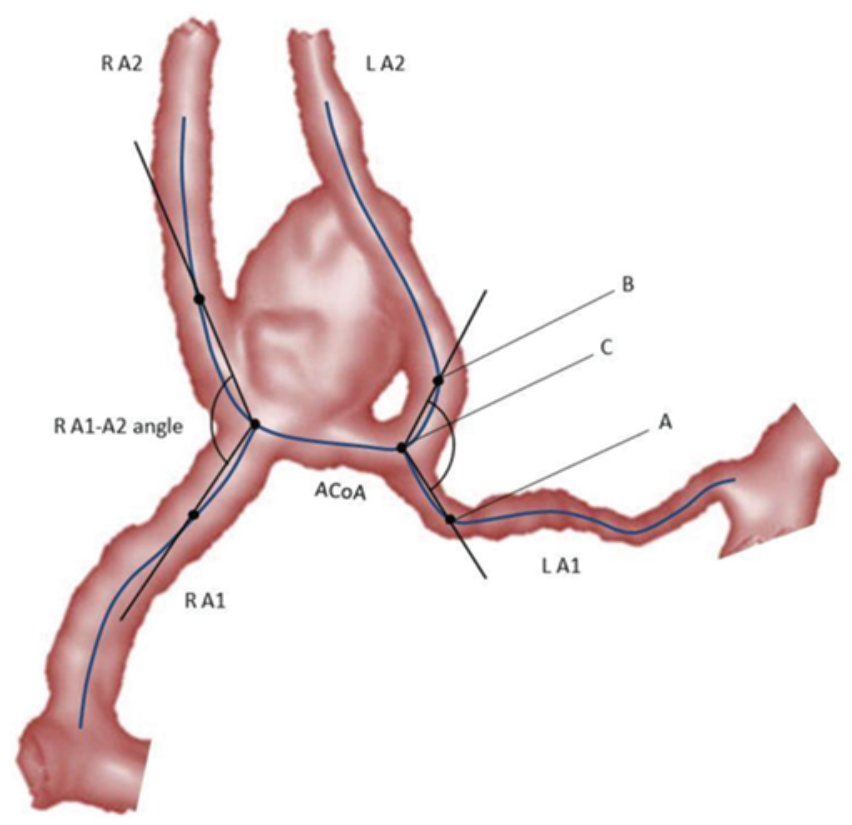

FIG. 2. Three-dimensional model of an $A C o A$ aneurysm, frontal view, showing the angular analysis of vessels from the anterior complex of the $C W$. The barycenter of the section of greatest curvature along the $A_{1}$ segment $(A)$, of greatest curvature along the $A_{2}$ segment $(B)$, and of the $A_{1}-A_{2}$ junction $(C)$ are shown. was described as "aplastic" when not detectable. This classification scheme and these measurements were based on the report by Kaspera et al. ${ }^{16}$

The $\mathrm{A}_{1}-\mathrm{A}_{2}$ angle measurements were performed with reconstructions from maximum intensity projection images by using the plane spanning the distal portion of $\mathrm{A}_{1}$ (from the greatest curvature) through the $A_{1}-A_{2}$ junction and the proximal portion of $\mathrm{A}_{2}$ (up to the greatest curvature). The arms of the angle were drawn from the points of greatest curvature along the $A_{1}$ and $A_{2}$ segments, located on the barycenter of the vessel. The apex of the angle was placed at the $A_{1}-A_{2}$ junction and on the barycenter of the vessel. When there was aplasia of the $\mathrm{A}_{1}$ segment, measurements were performed only on the contralateral segment.

We also measured the morphological parameters of the IA, including the major axis, the neck diameter, and its lateralization. The major axis was defined as the widest diameter on a cross-section of the IA, measured from the base of the IA. Lateralization of the IA was classified according to the side on which it grew and in relation to the $A_{1}-A_{2}$ junction. The $A_{1}-A_{2}$ angles were studied according to the side where the aneurysm was located. Given the lack of IAs in the HFDR group, we chose the narrower of the 2 angles for the purpose of conducting a comparative analysis of the 3 groups.

All measurements were performed by the same operator (C.L.).

\section{Statistical Analysis}

Descriptive analyses made it possible to compare the clinical and demographic characteristics of the 3 groups. Qualitative variables were tested using the chi-square test and Fisher's exact test. Quantitative variables were compared among the 3 groups with the Kruskal-Wallis test. Two-by-two comparisons were performed with the MannWhitney U-test. A Spearman correlation was used to analyze the correlation between 2 quantitative variables. The $\mathrm{p}<0.05$ threshold was considered significant. Statistical analyses were performed using SPSS software (SPSS Inc.).

\section{Results}

A total of 24, 20, and 24 individuals were included from the FACAA, HFDR, and SACAA groups, respectively. Clinical and demographic information is shown in Table 1 . The mean age overall was $53 \pm 13$ years. There was no difference in age $(p=0.58)$ or sex ratio $(p=0.22)$ among the 3 groups. Likewise, we found no significant difference among the 3 groups for cardiovascular risk factors, such as smoking $(\mathrm{p}=0.94)$, diabetes $(\mathrm{p}=0.90)$, and dyslipidemia $(\mathrm{p}=0.47)$.

Morphological characteristics of the aneurysms are shown in Table 2. The intrinsic characteristics of the IA (e.g., major axis, neck) were similar between the FACAA and SACAA groups. The VAC values were statistically different among the 3 groups $(20.5 \%$ vs $7.9 \%$ vs $14.7 \%$ for the FACAA, HFDR, and SACAA groups, respectively; $p=0.045)$ and favored more frequent asymmetrical formation in the FACAA group. The aneurysm-side $A_{1}-A_{2}$ angles were also different among the 3 groups. Indeed, these appeared lower in the groups of patients with aneu- 

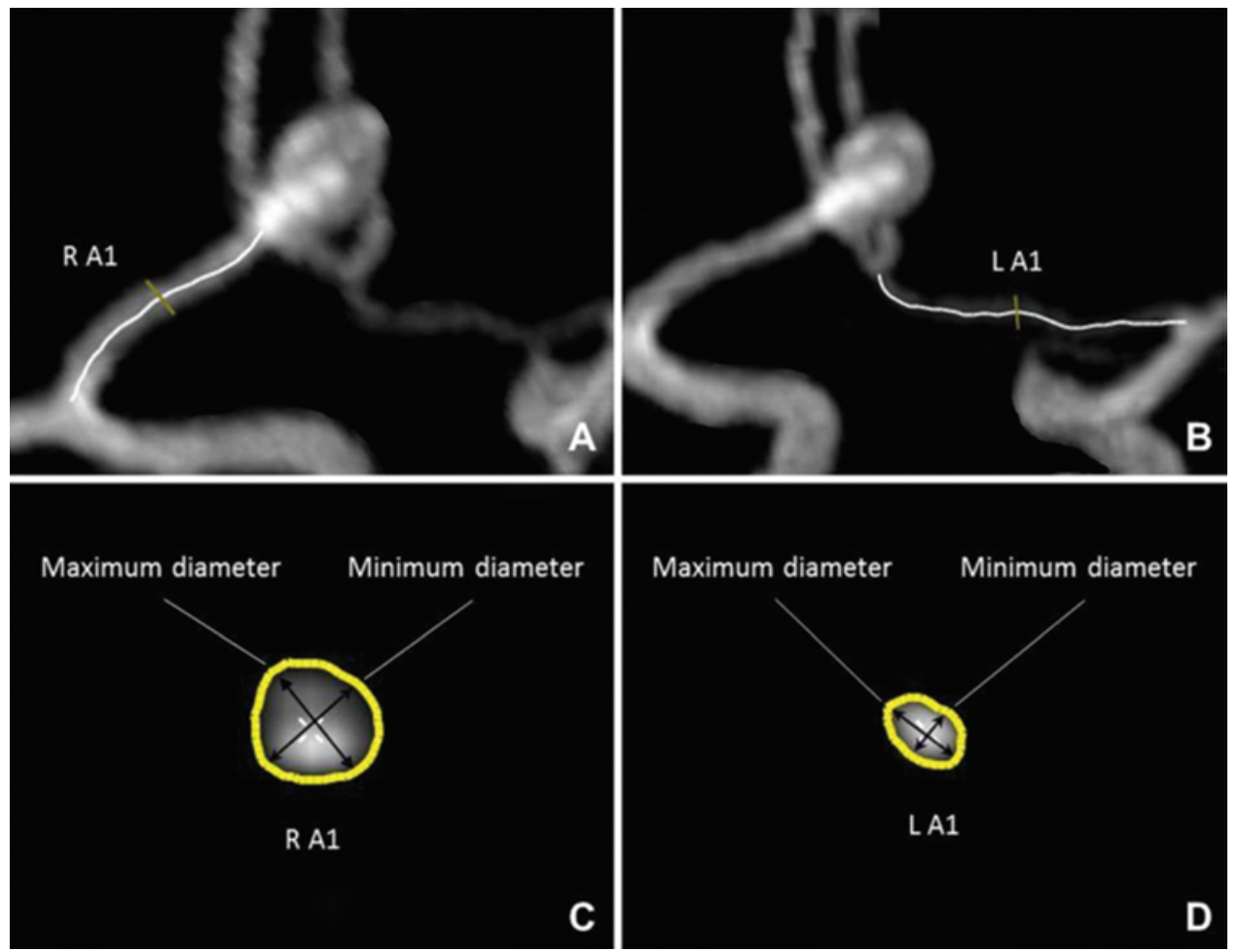

FIG. 3. Diameter measurements of the $A_{1}$ segment of the right and left anterior cerebral arteries.

rysms than in the HFDR group $\left(105.69^{\circ}\right.$ and $105.11^{\circ}$ for the FACAA and SACAA groups, respectively, vs $121.03^{\circ}$ for the HFDR group; $\mathrm{p}=0.005)$. On the other hand, the 3 groups had no difference in $\mathrm{A}_{1}-\mathrm{A}_{2}$ angle on the side opposite from the IA. In the FACAA and SACAA groups, there was no correlation between IA size and $A_{1}-A_{2}$ angle $(\mathrm{p}=0.55)$.

Paired comparisons between groups are reported in Table 3. The VAC was significantly higher in the FACAA group than in the HFDR group $(20.5 \%$ vs $7.9 \%$, respectively; $\mathrm{p}=0.002)$. Conversely, there was no significant difference in VAC values between the FACAA and SACAA groups $(p=0.17)$ or the SACAA and HFDR groups $(p=$ $0.15)$. The $A_{1}-A_{2}$ angles on the IA side were significantly lower for both IA groups than those in the HFDR group. Indeed, we found this difference between the FACAA and HFDR groups $\left(105.69^{\circ}\right.$ vs $121.03^{\circ}$, respectively; $\left.p=0.003\right)$ and between the SACAA and HFDR groups $\left(105.11^{\circ}\right.$ vs $121.03^{\circ}$, respectively; $\left.p=0.007\right)$. The $A_{1}-A_{2}$ angles on the aneurysm side were not different $(p=0.95)$ between the FACAA and SACAA groups.

\section{Discussion}

Our work shows that anatomical formation of the anterior complex in patients with FACAA was different from that in their HFDRs. It has been previously demonstrated that the geometry of the affected arteries can contribute to the formation of the IA, independent of familial contributions. 2,6-8,12,15,18,39 Specifically, the anatomy of the anterior complex of the $\mathrm{CW}$ can create a trend toward the formation of IAs in the ACoA. ${ }^{16}$ Indeed, IAs form preferentially at arterial bifurcations and on tight vascular curvatures (having a low radius of curvature). ${ }^{8}$ Recent studies investigating the anterior complex of the $\mathrm{CW}$ using $3 \mathrm{D}$ computed tomography angiography (CTA) models found that the incidence of ACoA IA is linked to $A_{1}-A_{2}$ angles and the asymmetry between the $\mathrm{A}_{1}$ segments. ${ }^{15,18}$ Kaspera et

TABLE 1. Clinical and demographic data for patients in the FACAA, HFDR, and SACAA groups

\begin{tabular}{lcccc}
\hline \multicolumn{1}{c}{ Parameter } & $\begin{array}{c}\text { FACAA } \\
\text { Group }\end{array}$ & $\begin{array}{c}\text { HFDR } \\
\text { Group }\end{array}$ & $\begin{array}{c}\text { SACAA } \\
\text { Group }\end{array}$ & $\begin{array}{c}\text { p } \\
\text { Value }\end{array}$ \\
\hline No. of patients & 24 & 20 & 24 & NA \\
\hline No. of women (\%) & $13(54)$ & $13(65)$ & $9(38)$ & $0.22^{*}$ \\
\hline Mean age in yrs (SD) & $54 \pm 13$ & $55 \pm 15$ & $51 \pm 11$ & $0.58 \dagger$ \\
\hline Smoking status, no. (\%) & & & & $0.94^{*}$ \\
\hline Still smoking & $6(25)$ & $6(30)$ & $6(25)$ & \\
\hline Quit smoking <3 yrs & $3(13)$ & $1(5)$ & $1(4)$ & \\
\hline Quit smoking >3 yrs & $4(17)$ & $5(25)$ & $1(4)$ & \\
\hline Never smoked & $8(33)$ & $8(40)$ & $9(38)$ & \\
\hline Alcohol consumption, no. (\%) & & & & $0.06^{*}$ \\
\hline$\quad \leq 150$ g/wk & $11(46)$ & $10(50)$ & $0(0)$ & \\
\hline$>150$ g/wk & $2(8)$ & $2(10)$ & $3(13)$ & \\
\hline High blood pressure, no. (\%) & $11(46)$ & $6(30)$ & $9(37)$ & $0.56^{*}$ \\
\hline Diabetes, no. (\%) & $1(4)$ & $0(0)$ & $0(0)$ & $0.90^{*}$ \\
\hline Dyslipidemia, no. (\%) & $6(25)$ & $6(30)$ & $3(13)$ & $0.47^{*}$ \\
\hline
\end{tabular}

$N A=$ not applicable.

* Chi-square test or Fisher's exact test.

$\dagger$ Kruskal-Wallis test. 
TABLE 2. Measurements of the anterior complex of the CW for patients in the FACAA, HFDR, and SACAA groups

\begin{tabular}{|c|c|c|c|c|}
\hline Parameter & FACAA & HFDR & SACAA & $\mathrm{p}$ Value \\
\hline No. of patients & 24 & 20 & 24 & NA \\
\hline Major axis of aneurysm in $\mathrm{mm}^{*}$ & $4.3(3.2-7)$ & & $5(4-6.4)$ & $0.41 \dagger$ \\
\hline Aneurysm neck in $\mathrm{mm}^{*}$ & $3(2-3.3)$ & & $3(2.2-3.3)$ & $0.51 \dagger$ \\
\hline Aneurysm side on It $\ddagger$ & $13(54 \%)$ & & $11(46 \%)$ & \\
\hline Total no. of aneurysms & & & & $0.19 \dagger$ \\
\hline 1 & $13(54 \%)$ & & $20(83 \%)$ & \\
\hline 2 & $7(29 \%)$ & & $3(13 \%)$ & \\
\hline 3 & $3(13 \%)$ & & $1(4 \%)$ & \\
\hline 4 & $1(4 \%)$ & & $0(0 \%)$ & \\
\hline VAC in \% & $20.5(10.6-29.5)$ & $7.9(5.2-15.6)$ & $14.7(7.2-21.1)$ & $0.045 \S$ \\
\hline Configuration of $A_{1}$ segments $\ddagger$ & & & & $0.03 \S$ \\
\hline Symmetry & $4(17 \%)$ & $12(60 \%)$ & $6(25 \%)$ & \\
\hline Asymmetry & $12(50 \%)$ & $8(40 \%)$ & $14(58 \%)$ & \\
\hline Hypoplasia & $4(17 \%)$ & $0(0 \%)$ & $0(0 \%)$ & \\
\hline Aplasia & $4(17 \%)$ & $0(0 \%)$ & $4(17 \%)$ & \\
\hline \multicolumn{5}{|l|}{$A_{1}-A_{2}$ angle in degrees ${ }^{*}$} \\
\hline Aneurysm side & 105.69 (95.46-116.98) & 121.03 (113.83-127.99) & $105.11(86.21-121.42)$ & $0.005 \S$ \\
\hline Side opposite from aneurysm & 113.51 (97.99-129.19) & 121.03 (113.83-127.99) & $120.80(94.65-129.61)$ & $0.57 \S$ \\
\hline
\end{tabular}

al. have shown that an $\mathrm{A}_{1}-\mathrm{A}_{2}$ angle $\leq 100^{\circ}$ and $\mathrm{A}_{1}$ segment asymmetry $>40 \%$ were the main risk factors associated with the development of an ACoA IA. ${ }^{16}$ Numerous studies using 3D fluid mechanics models have shown that an increase in the asymmetry of the $A_{1}$ segments would cause a significant increase in hemodynamic stress and wall-shear stress at the junction of the $A_{1}$ segment and the ACoA..$^{38,43}$ Abnormal wall-shear stress induces an endothelial response mediated by proinflammatory factors, from metalloproteinase activation to smooth muscle cell death to extracellular matrix degradation to vascular remodeling. ${ }^{14,19,28,29,32,41}$

Our results show that the angle of the $A_{1}-A_{2}$ segment is narrower in the FACAA group than in the HFDR group; furthermore, the FACAA group also had a higher VAC than the HFDR group. These findings suggest 2 hypotheses. The first is that, for patients with FACAA, the anatomical form of the anterior complex of the CW may be inherited and thus favor the clustering of IA cases within families. For patients in our study, this anatomical form would favor ACoA IA formation. Previous studies have shown cases of identical aneurysm location in members of the same family or in twins. ${ }^{1,3,17,25,27}$ Indeed, the Familial Intracranial Aneurysm (FIA) study reported a high agreement of aneurysm location among IA patients belonging to the same family. ${ }^{26}$ These observations may thus suggest that anatomical vulnerability to aneurysm formation is hereditary. The FIA study did not draw conclusions regarding ACOA IAs because this agreement was not observable for ACoA IA location in the FIA study. ${ }^{26}$ This hypothesis will be the subject of future work studying the CW in subsequent generations of families with IA.

The second hypothesis suggested by our results is that familial ACoA IA development may have no innate origin. Only hemodynamic factors are responsible for aneurysm development, and the anatomical form of the anterior complex of the $\mathrm{CW}$ would not be heritable. Hemodynamic forces would thus have a direct, mechanical effect on ACoA IA formation. Within the same family, ACoA IA formation could be related to other aneurysm locations (e.g., middle cerebral artery, posterior communicating artery, and so forth) that, on the other hand,

TABLE 3. Comparison of the VAC and $A_{1}-A_{2}$ angle on the aneurysm side for patients in the FACAA, HFDR, and SACAA groups *

\begin{tabular}{|c|c|c|c|c|c|c|}
\hline Parameter & FACAA & HFDR & SACAA & $\begin{array}{c}\text { FACAA vs } \\
\text { HFDR, } \\
\text { p Value }\end{array}$ & $\begin{array}{c}\text { FACAA vs } \\
\text { SACAA, } \\
\text { p Value }\end{array}$ & $\begin{array}{c}\text { SACAA vs } \\
\text { HFDR, } \\
\text { p Value }\end{array}$ \\
\hline VAC in \% & $20.5(10.6-29.5)$ & $7.9(5.2-15.6)$ & $14.7(7.2-21.1)$ & $0.002 \dagger$ & $0.17 \dagger$ & $0.15 \dagger$ \\
\hline$A_{1}-A_{2}$ angle on IA side (degrees) & $105.69(95.46-116.98)$ & $121.03(113.83-127.99)$ & $105.11(86.21-121.42)$ & $0.003 \dagger$ & $0.95 \dagger$ & $0.007 \dagger$ \\
\hline
\end{tabular}

\footnotetext{
* Values expressed as medians (interquartile range).

$\dagger$ Mann-Whitney U-test.
} 
may be genetically determined. However, it does seem that hereditary transmission of the $\mathrm{CW}$ shape occurs in animals. Mongolian gerbils were used as animal models for cerebral ischemia because of their spontaneous variations in the CW. ${ }^{23}$ Indeed, Du et al. noticed that the CW shapes in gerbils are transmittable from 1 generation to another, and that there is a close relationship between the anterior vascular complex of the mothers and that of their offspring. ${ }^{11,24}$ In generating transgenic mice in which the Notch signaling pathway was eliminated, Proweller et al. observed anomalies in the cerebrovascular structures of the mice, including interruption of the $\mathrm{CW}$, particularly within the anterior complex. ${ }^{33,44}$

However, given studies performed at a premature embryonic stage, it has also been suggested that anatomical variations in the $\mathrm{CW}$ are genetically determined in humans. ${ }^{9,30}$ Indeed, the frequency of different $\mathrm{CW}$ shapes varies according to ethnic group. ${ }^{10}$ But at this time, there is no evidence regarding any gene that may be linked with CW shape in humans.

MRA was the testing and measurement tool used for this study. Preexisting data confirm its validity as a tool for screening IAs. Indeed, there is excellent agreement among observers regarding its IA characterization..$^{20,27,42}$ In our study, experienced interventional neuroradiologists from each center phenotyped the IAs in order to exclusively identify patients with saccular bifurcation IA. The major asset of our study was having access to imaging data for each patient. This allowed us to analyze specific anatomical variations in patients with or without IAs.

Our study focused on IAs of the ACoA, but our strategy could be applied to all other bifurcation IA locations as well. Nonetheless, the clinical interest of our study is to help practitioners recognize the importance of carefully examining the shape of the $\mathrm{CW}$ in patients tested for IAs. Monitoring these patients will undoubtedly have to be tailored to whether the $\mathrm{CW}$ has any high-risk shape.

Our results suggest that, for patients with FACAA, hemodynamic factors are just as important as heritable factors if there is no link to these latter factors. More fundamentally, for studies of familial IA, the selection of candidate genes or pathways could be oriented toward the different developmental pathways of the CW. We suggest a novel approach to understanding the physiopathology of IAs. We also agree with the conclusions made by Lee et al. in their study of over 200 mirror-like IAs. ${ }^{22}$ They showed that the frequency of mirror-like IAs varies depending on the location, with mirror-like IAs being overrepresented among intracavernous IAs, for example. It suggests that different pathophysiological mechanisms may be at the root of the different aneurysm locations. Genome-wide association studies undoubtedly need to focus further on particular IA phenotypes.

Our work entailed certain limitations. To limit irradiation while also considering spatial resolution, we chose MRA for our GAIA protocol for testing familial forms of IA. To maximize comparability among the groups, the MRA images of the SACAA group were also chosen. In any case, most morphological studies of the same type as ours use CTA, whose spatial resolution is apparently better than MRA. This constitutes a limitation more applicable to measurement of the $A_{1}-A_{2}$ angles than to measurement of the $\mathrm{A}_{1}$ diameter ratios, which is, by definition, comparative and relative.

We did not observe any significant difference between the anatomical shapes of the anterior complex of the CW for patients in the FACAA or SACAA groups. This could be attributed to a lack of statistical power from the small number of patients. Furthermore, heritability cannot be totally excluded in sporadic cases. Likewise, among the HFDRs, certain individuals with small aneurysms could have been unidentified and thus not properly classified. Moreover, although the HFDR group members did not present with any IAs during the study period, we cannot rule out the possibility of an IA developing later on.

Another limitation was our inclusion of patients with previously formed IA. Consequently, hemodynamic parameters at the start of IA formation may have been slightly different from those measured during the time of the study. At this time, no study has shown how the presence of an IA can influence affected arteries. However, in the FACAA and SACAA groups, there was no correlation between IA size and $\mathrm{A}_{1}-\mathrm{A}_{2}$ angle.

The selection of the SACAA and HFDR groups constitutes a limitation of this study. We made an effort to recruit patients with SACAA as they came into our medical center from among the patients listed as having IA.

\section{Conclusions}

Our work points out the importance of the anatomical shape of the anterior complex of the CW in ACoA IA formation. In particular, we have shown that, even within the context of familial IA, the angular $\left(\mathrm{A}_{1}-\mathrm{A}_{2}\right)$ shapes and asymmetry of the $A_{1}$ segments are associated with the presence of ACoA IA. This leads us to raise 2 novel hypotheses: either susceptibility to IA formation is linked to anatomical shape inherited within a given family, or within the particular context of the ACoA, IA formation is not inherited and is mostly induced by hemodynamic constraints. Either hypothesis should be verified by studying larger samples and more IA locations.

\section{Acknowledgments}

We thank Hervé Le Marec, Stéphanie Chatel, Emmanuelle Bourcereau, and Gervaise Loirand for their contributions to the organization of the GAIA project (INSERM, UMR1087, l'institut du thorax, CHU de Nantes). We also thank the GAIA investigators for their contributions to patient recruitment: Anne Pasco (University Hospital of Angers); Stéphane Velasco and Samy Boucebci (University Hospital of Poitiers); Denis Herbreteau and Richard Bibi (University Hospital of Tours); Jean Christophe Ferre and Hélène Raoult (University Hospital of Rennes); Sophie Gallas (University Hospital of Créteil); Jérôme Berge and Xavier Barreau (University Hospital of Bordeaux); and the Genavie Foundation.

\section{References}

1. Acosta-Rua GJ: Familial incidence of ruptured intracranial aneurysms. Report of 12 cases. Arch Neurol 35:675-677, 1978

2. Baharoglu MI, Lauric A, Safain MG, Hippelheuser J, Wu C, Malek AM: Widening and high inclination of the middle 
cerebral artery bifurcation are associated with presence of aneurysms. Stroke 45:2649-2655, 2014

3. Bannerman RM, Ingall GB, Graf CJ: The familial occurrence of intracranial aneurysms. Neurology 20:283-292, 1970

4. Bourcier R, Redon R, Desal H: Genetic investigations on intracranial aneurysm: update and perspectives. J Neuroradiol 42:67-71, 2015

5. Broderick JP, Brott T, Tomsick T, Huster G, Miller R: The risk of subarachnoid and intracerebral hemorrhages in blacks as compared with whites. N Engl J Med 326:733-736, 1992

6. Can A, Ho AL, Dammers R, Dirven CMF, Du R: Morphological parameters associated with middle cerebral artery aneurysms. Neurosurgery 76:721-727, 2015

7. Can A, Ho AL, Emmer BJ, Dammers R, Dirven CMF, Du R: Association between vascular anatomy and posterior communicating artery aneurysms. World Neurosurg 84:1251-1255, 2015

8. Can A, Mouminah A, Ho AL, Du R: Effect of vascular anatomy on the formation of basilar tip aneurysms. Neurosurgery 76:62-66, 2015

9. Crompton MR: The pathology of ruptured middle-cerebral aneurysms with special reference to the differences between the sexes. Lancet 2:421-425, 1962

10. De Silva KRD, Silva R, Gunasekera WSL, Jayesekera RW: Prevalence of typical circle of Willis and the variation in the anterior communicating artery: A study of a Sri Lankan population. Ann Indian Acad Neurol 12:157-161, 2009

11. Du XY, Zhu XD, Dong G, Lu J, Wang Y, Zeng L, et al: Characteristics of circle of Willis variations in the Mongolian gerbil and a newly established ischemia-prone gerbil group. ILAR J 52:E1-E7, 2011

12. Endo S, Furuichi S, Takaba M, Hirashima Y, Nishijima M, Takaku A: Clinical study of enlarged infundibular dilation of the origin of the posterior communicating artery. J Neurosurg 83:421-425, 1995

13. Francis SE, Tu J, Qian Y, Avolio AP: A combination of genetic, molecular and haemodynamic risk factors contributes to the formation, enlargement and rupture of brain aneurysms. J Clin Neurosci 20:912-918, 2013

14. Frösen J, Tulamo R, Paetau A, Laaksamo E, Korja M, Laakso A, et al: Saccular intracranial aneurysm: pathology and mechanisms. Acta Neuropathol 123:773-786, 2012

15. Ingebrigtsen $\mathrm{T}$, Morgan MK, Faulder K, Ingebrigtsen L, Sparr T, Schirmer H: Bifurcation geometry and the presence of cerebral artery aneurysms. J Neurosurg 101:108-113, 2004

16. Kaspera W, Ładziński P, Larysz P, Hebda A, Ptaszkiewicz K, Kopera M, et al: Morphological, hemodynamic, and clinical independent risk factors for anterior communicating artery aneurysms. Stroke 45:2906-2911, 2014

17. Kasuya H, Onda H, Takeshita M, Hori T, Takakura K: Clinical features of intracranial aneurysms in siblings. Neurosurgery 46:1301-1306, 2000

18. Kasuya H, Shimizu T, Nakaya K, Sasahara A, Hori T, Takakura K: Angles between A1 and A2 segments of the anterior cerebral artery visualized by three-dimensional computed tomographic angiography and association of anterior communicating artery aneurysms. Neurosurgery 45:89-94, 1999

19. Kolega J, Gao L, Mandelbaum M, Mocco J, Siddiqui AH, Natarajan SK, et al: Cellular and molecular responses of the basilar terminus to hemodynamics during intracranial aneurysm initiation in a rabbit model. J Vasc Res 48:429-442, 2011

20. Korogi Y, Takahashi M, Mabuchi N, Miki H, Fujiwara S, Horikawa Y, et al: Intracranial aneurysms: diagnostic accuracy of three-dimensional, Fourier transform, time-of-flight MR angiography. Radiology 193:181-186, 1994
21. Krischek B, Inoue I: The genetics of intracranial aneurysms. J Hum Genet 51:587-594, 2006

22. Lee YJ, Parreira T, Matouk CC, Menezes R, Mandell DM, terBrugge KG, et al: Clinical characteristics and preferential location of intracranial mirror aneurysms: a comparison with non-mirror multiple and single aneurysms. Neuroradiology 57:35-40, 2015

23. Levine S, Payan H: Effects of ischemia and other procedures on the brain and retina of the gerbil (Meriones unguiculatus). Exp Neurol 16:255-262, 1966

24. Li Z, Huo X, Zhang S, Lu J, Li C, Guo M, et al: Selection of genes associated with variations in the Circle of Willis in gerbils using suppression subtractive hybridization. PLoS One 10:e0127355, 2015

25. Lozano AM, Leblanc R: Familial intracranial aneurysms. J Neurosurg 66:522-528, 1987

26. Mackey J, Brown RD Jr, Moomaw CJ, Hornung R, Sauerbeck L, Woo D, et al: Familial intracranial aneurysms: is anatomic vulnerability heritable? Stroke 44:38-42, 2013

27. Maeder PP, Meuli RA, de Tribolet N: Three-dimensional volume rendering for magnetic resonance angiography in the screening and preoperative workup of intracranial aneurysms. J Neurosurg 85:1050-1055, 1996

28. Malek AM, Alper SL, Izumo S: Hemodynamic shear stress and its role in atherosclerosis. JAMA 282:2035-2042, 1999

29. Metaxa E, Tremmel M, Natarajan SK, Xiang J, Paluch RA, Mandelbaum M, et al: Characterization of critical hemodynamics contributing to aneurysmal remodeling at the basilar terminus in a rabbit model. Stroke 41:1774-1782, 2010

30. Milenković Z, Vucetić R, Puzić M: Asymmetry and anomalies of the circle of Willis in fetal brain. Microsurgical study and functional remarks. Surg Neurol 24:563-570, 1985

31. Morita A, Fujiwara S, Hashi K, Ohtsu H, Kirino T: Risk of rupture associated with intact cerebral aneurysms in the Japanese population: a systematic review of the literature from Japan. J Neurosurg 102:601-606, 2005

32. Ota R, Kurihara C, Tsou TL, Young WL, Yeghiazarians Y, Chang M, et al: Roles of matrix metalloproteinases in flowinduced outward vascular remodeling. J Cereb Blood Flow Metab 29:1547-1558, 2009

33. Proweller A, Wright AC, Horng D, Cheng L, Lu MM, Lepore $\mathrm{JJ}$, et al: Notch signaling in vascular smooth muscle cells is required to pattern the cerebral vasculature. Proc Natl Acad Sci U S A 104:16275-16280, 2007

34. Rinkel GJ, Djibuti M, Algra A, van Gijn J: Prevalence and risk of rupture of intracranial aneurysms: a systematic review. Stroke 29:251-256, 1998

35. Ruigrok YM, Rinkel GJE: Genetics of intracranial aneurysms. Stroke 39:1049-1055, 2008

36. Ruigrok YM, Rinkel GJE, Wijmenga C: Genetics of intracranial aneurysms. Lancet Neurol 4:179-189, 2005

37. ter Laan M, Kerstjens-Frederikse WS, Metzemaekers JDM, van Dijk JMC, Groen RJM: Concordant symptomatic intracranial aneurysm in a monozygotic twin: a case report and review of the literature. Twin Res Hum Genet 12:295-300, 2009

38. Ujiie H, Liepsch DW, Goetz M, Yamaguchi R, Yonetani H, Takakura K: Hemodynamic study of the anterior communicating artery. Stroke 27:2086-2094, 1996

39. VanderArk GD, Kempe LC: Classification of anterior communicating aneurysms as a basis for surgical approach. J Neurosurg 32:300-303, 1970

40. Vlak MH, Algra A, Brandenburg R, Rinkel GJ: Prevalence of unruptured intracranial aneurysms, with emphasis on sex, age, comorbidity, country, and time period: a systematic review and meta-analysis. Lancet Neurol 10:626-636, 2011

41. Wang Z, Kolega J, Hoi Y, Gao L, Swartz DD, Levy EI, et al: Molecular alterations associated with aneurysmal remodeling 
are localized in the high hemodynamic stress region of a created carotid bifurcation. Neurosurgery 65:169-178, 2009

42. Wilcock D, Jaspan T, Holland I, Cherryman G, Worthington $\mathrm{B}$ : Comparison of magnetic resonance angiography with conventional angiography in the detection of intracranial aneurysms in patients presenting with subarachnoid haemorrhage. Clin Radiol 51:330-334, 1996

43. Xu L, Zhang F, Wang H, Yu Y: Contribution of the hemodynamics of A1 dysplasia or hypoplasia to anterior communicating artery aneurysms: a 3-dimensional numerical simulation study. J Comput Assist Tomogr 36:421-426, 2012

44. Yang K, Banerjee S, Proweller A: Regulation of pre-natal circle of Willis assembly by vascular smooth muscle Notch signaling. Dev Biol 381:107-120, 2013

\section{Disclosures}

The authors report no conflict of interest concerning the materi- als or methods used in this study or the findings specified in this paper.

\section{Author Contributions}

Conception and design: Bourcier. Acquisition of data: Bourcier, Lenoble. Analysis and interpretation of data: Bourcier, Lenoble, Guyomarch-Delasalle. Drafting the article: Bourcier, Lenoble, Desal. Critically revising the article: Bourcier, Daumas-Duport, Papagiannaki, Redon, Desal. Approved the final version of the manuscript on behalf of all authors: Bourcier. Statistical analysis: Guyomarch-Delasalle. Study supervision: Bourcier.

\section{Correspondence}

Romain Bourcier, Department of Diagnostic and Interventional Neuroradiology, CHU Nantes HGRL, Nantes F-44000, France. email: romain.bourcier2@gmail.com. 